\title{
Lessons of the month 2: A case of Behçet's disease: 70\% have ophthalmic involvement
}

\author{
Authors: Ishna Auluck, ${ }^{A}$ Ayesha Karimi ${ }^{B}$ and Simon Taylor ${ }^{C}$
}

\begin{abstract}
A 34-year-old man presented to the emergency department with acute painless loss of vision of the left eye. Past medical history included painful lumps in the legs and frequent mouth ulcers, which were undiagnosed. The patient's visual acuity was $6 / 5$ and counting fingers in the right and left eye, respectively. There were extensive intraretinal haemorrhages and venous sheathing in the superior quadrant of the left eye with associated disc oedema. The case was discussed in a multidisciplinary team meeting in the presence of ophthalmology, dermatology and immunology and a diagnosis of Behçet's disease was reached. The patient was commenced on intravenous methylprednisolone for 3 days followed by a switch to oral prednisolone. Due to recalcitrant uveitis, an intravitreal dexamethasone implant was administered. Eventually, systemic azathioprine and infliximab were commenced with frequent review by ophthalmology and immunology. The macular oedema improved but, unfortunately, the patient's visual acuity did not recover. Behçet's disease is a complex vasculitis involving multiple organ systems. Ocular manifestations can occur in $70 \%$ of patients, comprising retinal vasculitis, anterior uveitis, iridocyclitis, chorioretinitis, scleritis, keratitis, vitreous haemorrhage, optic neuritis, conjunctivitis, retinal vein occlusion and retinal neovascularisation. A tailored multidisciplinary approach is required, with corticosteroids being the mainstay of treatment.
\end{abstract}

KEYWORDS: Behçet's, ophthalmology, rheumatology, immunology, autoimmune

\section{Introduction}

Behçet's disease (BD) was first described in 1937 by Turkish dermatologist and scientist, Hulusi Behçet, as a classic tri-symptom complex of hypopyon, iritis and orogenital aphthosis. ${ }^{1}$ We now know $\mathrm{BD}$ as a chronic, relapsing-remitting, occlusive vasculitis with multi-organ involvement including aphthous ulceration of the oral mucous membrane, genital ulceration, uveitis, erythema nodosum,

Authors: Afoundation doctor, St Peter's Hospital, Chertsey, UK; Bophthalmology registrar, Royal Surrey County Hospital, Guildford, UK; ' ophthalmology consultant, Royal Surrey County Hospital, Guildford, UK arthritis, thrombophlebitis, colitis and neurological disturbances. ${ }^{2,3}$ Histologically, there is a combination of perivascular neutrophilic or lymphocytic infiltration, endothelial cell damage, coupled with a prothrombotic tendency. ${ }^{4-6}$ The prevalence is mainly in the Mediterranean basin and there is a prominent similarity of the distribution to the ancient Silk Road, suggesting that an inherited tendency to develop BD was spread by merchants who travelled these trading routes. ${ }^{7-9} \mathrm{BD}$ is more predominant in males in their 2nd to 4 th decade of life. ${ }^{10,11}$ Currently the cause of $B D$ is unknown but it is believed that immunogenetics, immune regulation, vascular abnormalities or bacterial and viral infections may play a role. $^{12-19}$

\section{Ocular manifestations}

Ocular manifestations can occur in $70 \%$ of patients. ${ }^{20}$ The most visually affecting presentation is that of retinal vasculitis. ${ }^{21}$ Other ocular presentations include anterior uveitis, iridocyclitis, chorioretinitis, scleritis, keratitis, vitreous haemorrhage, optic neuritis, conjunctivitis, retinal vein occlusion and retinal neovascularisation. ${ }^{22}$ Decreased visual acuity is a result of retinal vasculitis, macular oedema, secondary glaucoma and cataracts. ${ }^{23}$ The Japanese Ministry of Health, Labour and Welfare's Behcet's Disease Research Committee previously stated that individuals with ocular involvement would have a visual acuity of less than 0.1 at 8 years; with $40 \%$ of cases leading to blindness in about 10 years. ${ }^{24,25}$ However, visual prognosis has been improving with the recent moderation of $\mathrm{BD}$ and development of new therapies.

\section{Case presentation}

A 34-year-old man presented to the emergency department with acute painless loss of vision of the left eye. Past medical history included painful lumps in the legs and frequent mouth ulcers, which was undiagnosed.

On ocular examination the patient's visual acuity was $6 / 5$ in the right eye and counting fingers in the left eye. The anterior segments were unremarkable bilaterally with intraocular pressures of 13 to 14 $\mathrm{mmHg}$ in each eye. Fundal examination of the right eye was normal (Figs 1a and b). There were extensive intraretinal haemorrhages and venous sheathing in the superior quadrant of the left eye with associated disc oedema (Figs 1c and d). Optical coherence tomography showed macular oedema of the left eye (Fig 2).

The case was discussed in a multidisciplinary team meeting in the presence of ophthalmology, dermatology and immunology and a diagnosis of $\mathrm{BD}$ was reached. The patient was started on 
Fig 1. Fundal photograph of the a) right eye's optic disc, b) right eye's macula c) left eye's optic disc and d) left eye's macula showing extensive retinal haemorrhages and venous sheathing in the superior quadrant

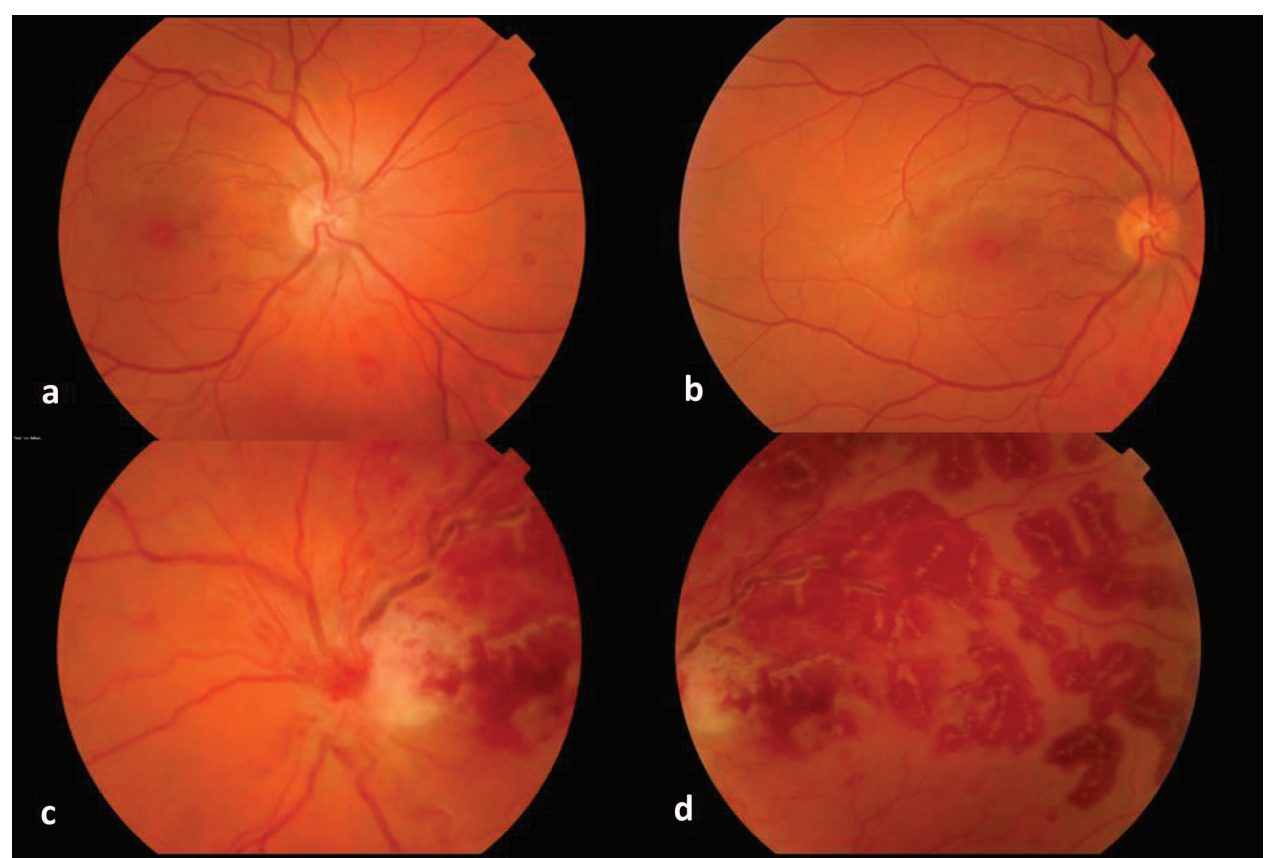

intravenous methylprednisolone for 3 days followed by a switch to oral prednisolone. Due to recalcitrant uveitis, an intravitreal dexamethasone implant was administered. Eventually, systemic azathioprine and infliximab were commenced with frequent review by ophthalmology and immunology. The macular oedema improved but, unfortunately, the patient's visual acuity did not recover (Fig 3).

\section{Management}

$B D$ requires a multidisciplinary approach, with input from dermatology for the evaluation of mucocutaneous lesions; ophthalmology for ocular involvement; rheumatology or orthopaedics for evaluation of joint involvement; neurology and psychiatry for central nervous system (CNS) involvement; internal medical specialists for evaluation of gastrointestinal (GI), pulmonary, renal or endocrine disorders; general surgeons for evaluation of GI tract involvement; cardiologists for cardiovascular disease and ear, nose and throat or dental specialists for evaluating the oral cavity. Treatment of BD is symptomatic and empirical, ie the choice of treatment depends on the site and severity of clinical manifestations. Corticosteroids are the mainstay for all clinical features. ${ }^{26}$ They are beneficial
Fig 2. Optical coherence tomography of the a) right eye and b) left eye showing macular oedema.

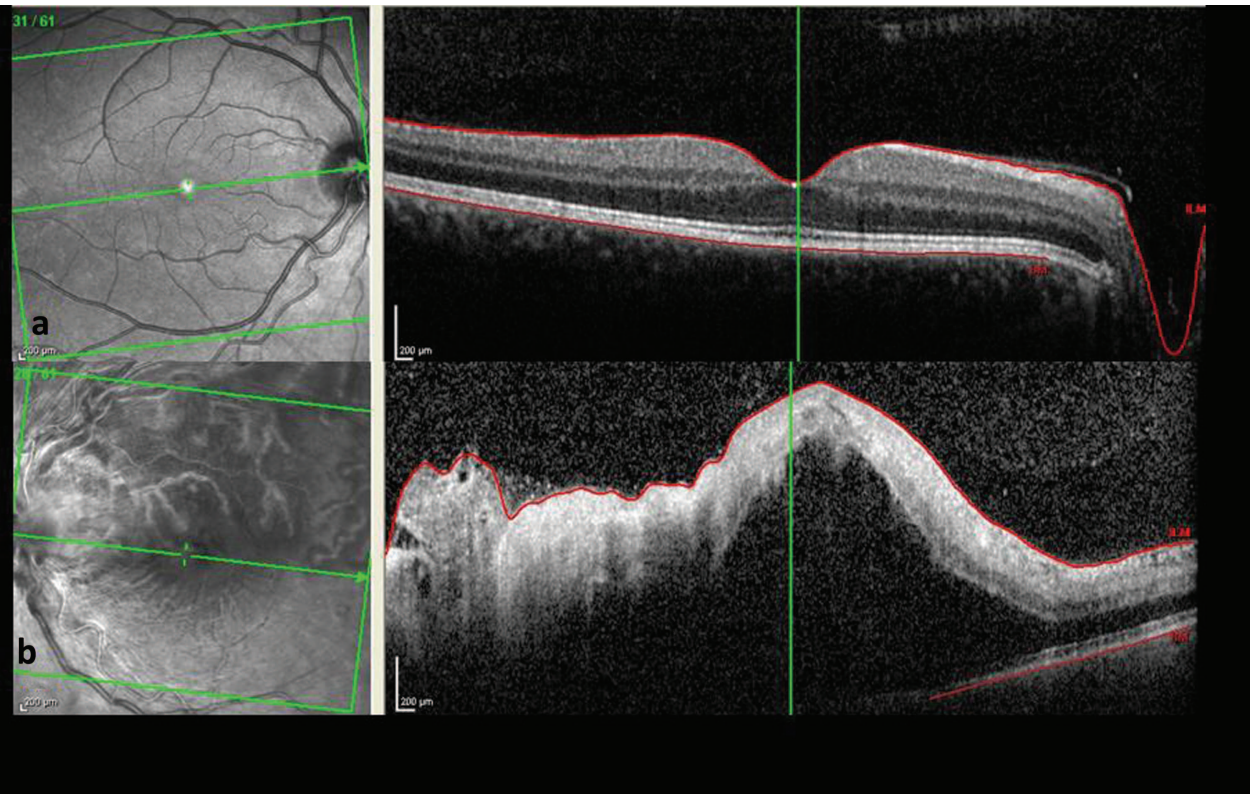




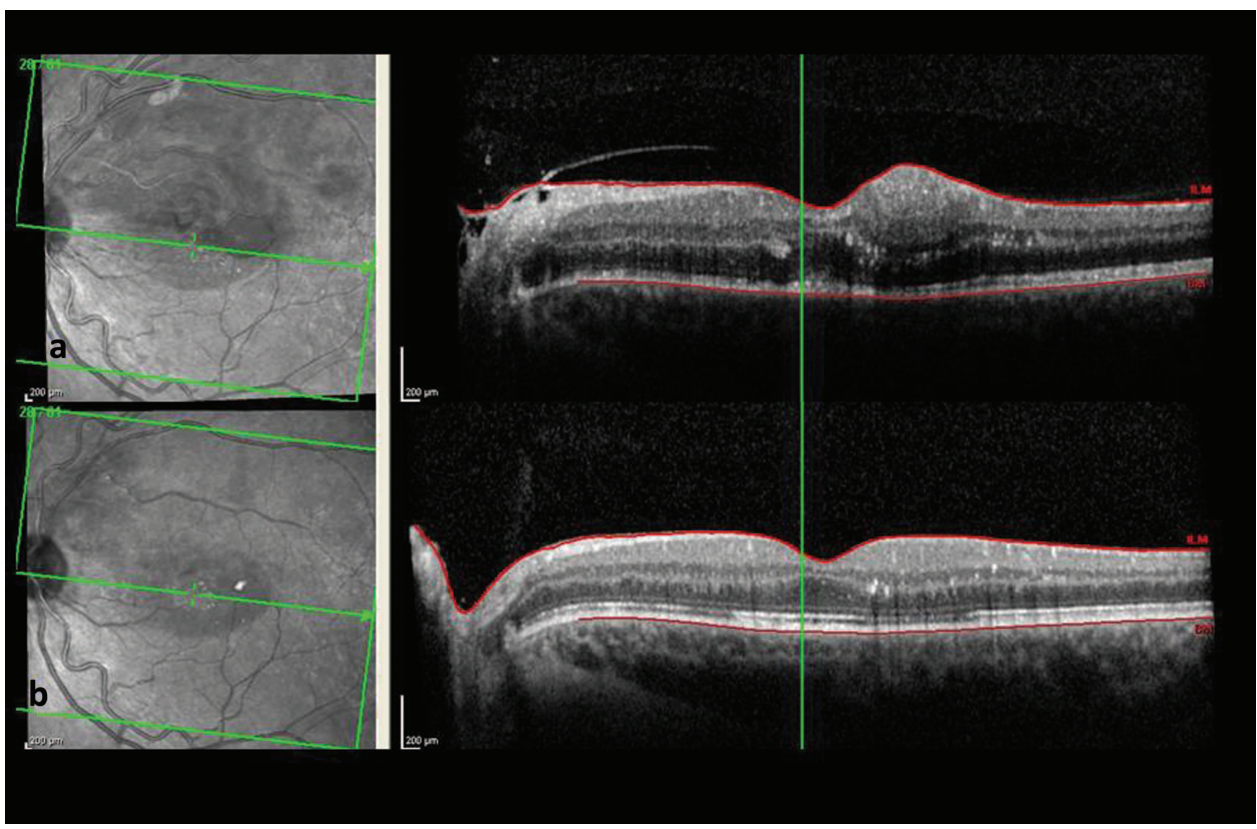

Fig 3. Optical coherence tomography of the left eye a) after initiating treatment and b) at completion of steroids and immunotherapy.

in the acute manifestations of $\mathrm{BD}$, but there is no definite evidence in the effectiveness in controlling disease progression. Importantly, adverse effects of long-term therapy with corticosteroids must be considered. Other treatment modalities do exist. Mucocutaneous lesions and arthritis can be treated with non-steroidal anti-inflammatory drugs, zinc sulphate, levamisole, colchicine, dapsone or immunosuppressive therapies, including azathioprine, chlorambucil or cyclophosphamide. ${ }^{27}$ Uveitis and CNS involvement can be managed by azathioprine, cyclosporine or methotrexate. ${ }^{28}$ For patients who develop vascular complications and thrombosis, an important consideration is appropriate anticoagulation. Other therapeutic approaches that have been trialled include interferon (IFN) alfa, IFN gamma, acyclovir depending on patient response and tolerance to adverse effects. ${ }^{29,30}$

\section{Summary}

$\mathrm{BD}$ is a complex vasculitis involving multiple organ systems. Ocular manifestations are common. A tailored multidisciplinary approach is required in the management, with corticosteroids being the mainstay of treatment.

\section{Key messages}

> Behçet's disease often involves input from immunology and rheumatology, however ocular manifestations can occur in $70 \%$ of patients, with visually significant manifestations if diagnosed late, so input from ophthalmology should be sought early.

$>$ We describe and provide images of a case of Behçet's uveitis.

$>$ We explore management options and how an intravitreal steroid implant improved retinal oedema.

> This case signifies the systemic associations of Behçet's disease and emphasises the importance of a tailored multidisciplinary approach in disease management.

\section{References}

1 Behçet H. Über rezidivierende, aphtöse, durch ein Virus verursachte Geschwüre am Mund, am Auge und an den Genitalien. Dermatologische Wochenschrift 1937;105:1152-63.

2 Ball G, Fessler B, Bridges Jr S (eds). Oxford textbook of vasculitis, 3rd edn. Oxford: Oxford University Press, 2014.

3 Gündüz Ö. Histopathological evaluation of Behçet's disease and identification of new skin lesions. Path Res Int 2012;2012:209316.

4 Kaneko F, Takahashi Y, Muramatsu R et al. Natural killer cell numbers and function in peripheral lymphoid cells in Behçet's disease. Br J Dermatol 1985;113:303-12.

5 Alpsoy E, Zouboulis C, Ehrlich G. Mucocutaneous lesions of Behçet's disease. Yonsei Med J 2007;48:573-85.

6 Calamia K, Schirmer M, Melikoğlu M. Major vessel involvement in Behçet's disease: an update. Curr Opin Rheumatol 2011;23:24-31.

7 Keino H, Okada A. Behçet's disease: global epidemiology of an Old Silk Road disease. Br J Ophthalmol 2007:91:1573-4.

8 Verity D, Marr J, Ohno S et al. Behçet's disease, the Silk Road and HLA-B51: historical and geographical perspectives. Tissue Antigens 1999;54:213-20.

9 Saadoun D, Wechsler B. Behçet's disease. Orphanet J Rare Dis 2012;7:1-6.

10 Bang $\mathrm{D}, \mathrm{Oh} \mathrm{S}$, Lee $\mathrm{K}$ et al. Influence of sex on patients with Behçet's disease in Korea. J Korean Med Sci 2003;18:231-5.

11 Zouboulis C, Kotter I, Djawari D et al. Epidemiological features of Adamantiades-Behçet's disease in Germany and in Europe. Yonsei Med J 1997:38:411-22.

12 Yazici H, Fresko I, Yurdakul S. Behçet's syndrome: disease manifestations, management, and advances in treatment. Nat Clin Pract Rheumatol 2007:3:148-55.

13 Androudi S. Current concepts in the etiology and treatment of Behçet disease. Surv Ophthalmol 2006;51:174.

14 Adam B, Çalikoglu E. Serum interleukin-6, procalcitonin and C-reactive protein levels in subjects with active Behçet's disease. J Eur Acad Dermatol Venereol 2004:18:318-20.

15 Bardak Y, Aridogan B. The demonstration of serum interleukin 6-8, tumor necrosis factor-alpha, complement, and immunoglobulin levels in Behçet's disease with ocular involvement. Ocul Immunol Inflamm 2004;12:53-8. 
16 Boyd S, Young S, Lightman S. Immunopathology of the noninfectious posterior and intermediate uveitides. Surv Ophthalmol 2001;46:209-33.

17 Erdem F, Gündogdu M, Kiki I et al. Vascular endothelial and basic fibroblast growth factor serum levels in patients with Behçet's disease. Rheumatol Int 2004;25:599-603.

18 Avci O, Ellidokuz E, Simşek I et al. Helicobacter pylori and Behçet's disease. Dermatology 1999;199:140-3.

19 Kiraz S, Oztürk M, Ertenli I et al. Parvovirus B19 infection in Behçet's disease. Ann Rheum Dis 2001;60:814-5.

20 Sakane T, Takeno M, Suzuki N et al. Behçet's disease. N Engl J Med 1999;341:1284-91.

21 Paovic J, Paovic P, Sredovic V. Behçet's disease: systemic and ocular manifestations. Biomed Res Int 2013:2013:247345.

22 Colvard M, Robertson D, O'Duffy D. The ocular manifestations of Behçet's disease. Arch Ophthalmol 1977:95:1813-7.

23 Taylor S, Singh J, Menezo V et al. Behçet disease: visual prognosis and factors influencing the development of visual loss. Am J Ophthalmol 2011:152:1059-66.

24 Hayashi T, Mizuki N. Ocular manifestations in Behçet's disease. Japan Med Assoc J 2006:49:260-8.
25 Nakae K, Hashimoto T, Inaba G et al. Results of a national epidemiological survey of patients with Behçet's disease (second report): relationship between clinical epidemiological results and HLA-B51. Behçet's Disease Research Committee, Japanese Ministry of Health and Welfare, Collected Papers 1992 1993:70-82.

26 McNally T, Damato E, Murray E et al. An update on the use of biologic therapies in the management of uveitis in Behçet's disease: a comprehensive review Orphanet J Rare Dis 2017;12:130.

27 Alpsoy E. New evidence-based treatment approach in Behçet's disease. Pathology Research International 2012;2012:871019.

28 Saleh Z, Arayssi T. Update on the therapy of Behçet disease. Ther Adv Chronic Dis 2014;5:112-34.

29 Kötter I, Günaydin I, Zierhut M, Stübiger N. The use of interferon alpha in Behçet disease: review of the literature. Semin Arthritis Rheum 2004;33:320-35.

30 Bonnet M, Ouzan D, Trepo C. [Plasma exchange and acyclovir in Behçet's disease]. J Fr Ophtalmol 1986;9:15-22.

Address for correspondence: Dr Ayesha Karimi, Royal Surrey County Hospital, Egerton Road, Guildford, Surrey GU2 7XX, UK. Email: ayeshakarimi@nhs.net

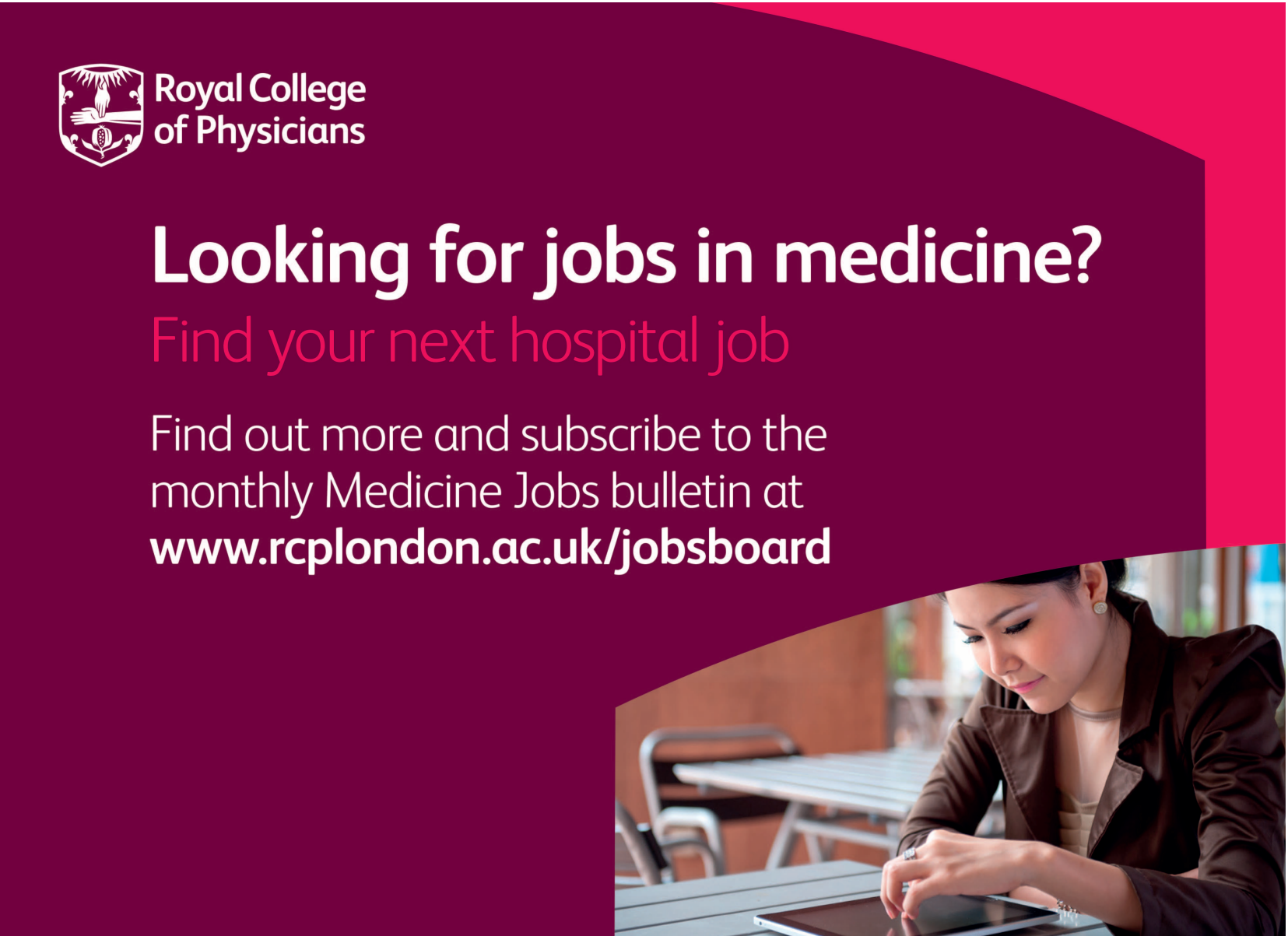

